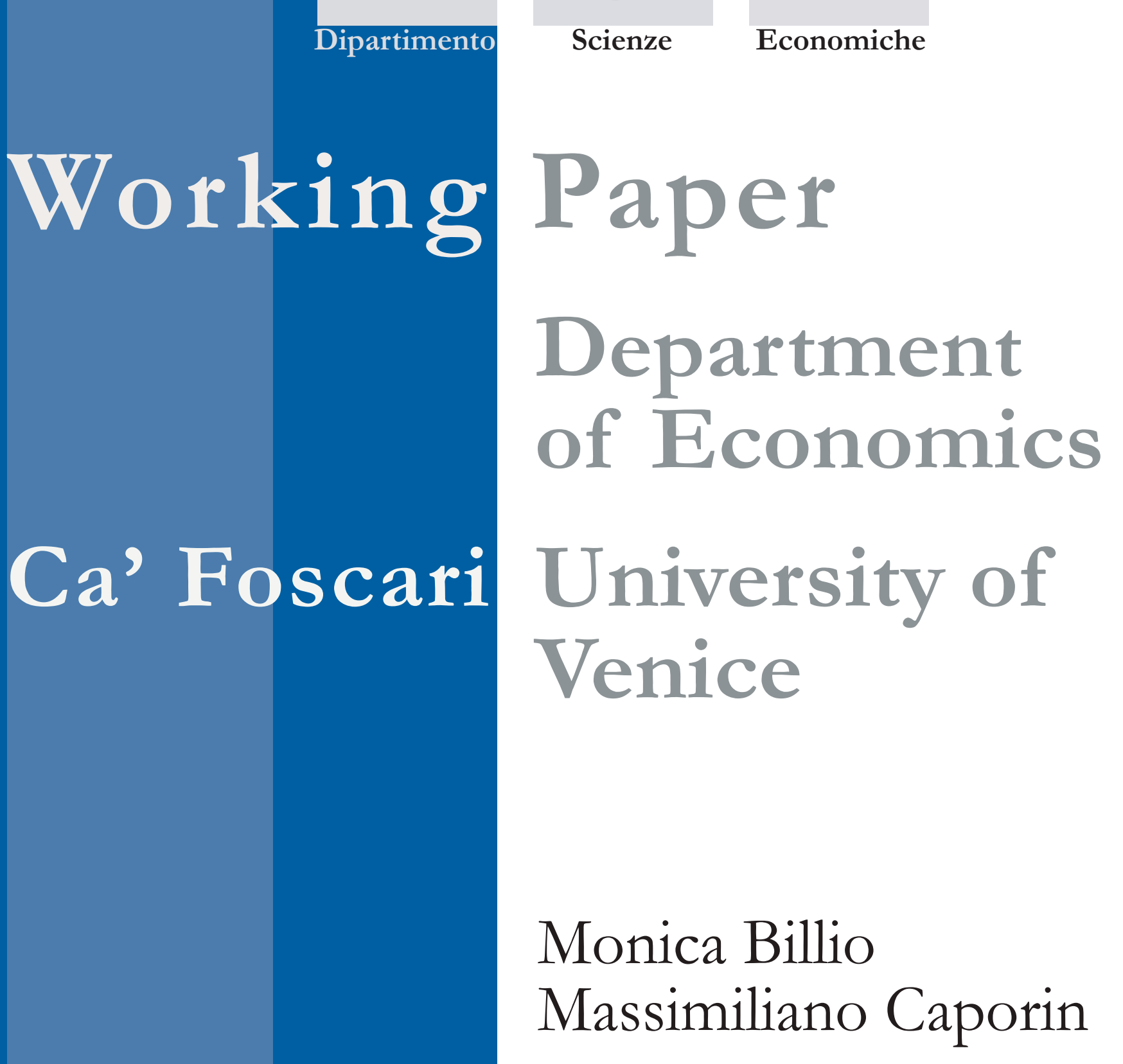




\title{
A generalized Dynamic Conditional Correlation model for portfolio risk evaluation
}

\author{
Monica Billio \\ University of $V$ enice
}

\author{
Massimiliano Caporin \\ University of Padova
}

\begin{abstract}
We propose a generalization of the Dynamic Conditional Correlation multivariate GARCH model of Engle (2002) and of the Asymmetric Dynamic Conditional Correlation model of Cappiello et al. (2006). The model we propose introduces a block structure in parameter matrices that allows for interdependence with a reduced number of parameters. Our model nests the Flexible Dynamic Conditional Correlation model of Billio et al. (2006) and is named Quadratic Flexible Dynamic Conditional Correlation Multivariate GARCH. In the paper, we provide conditions for positive definiteness of the conditional correlations. We also present an empirical application to the Italian stock market comparing alternative correlation models for portfolio risk evaluation.
\end{abstract}

\section{Keywords}

Dynamic correlations, Block-structures, Flexible correlation models

\section{JEL Codes}

C51, C32, G18

\author{
Address for correspondence: \\ Monica Billio \\ Department of Economics \\ Ca’ Foscari University of Venice \\ Cannaregio 873, Fondamenta S.Giobbe \\ 30121 Venezia - Italy \\ Phone: (++39) 0412349170 \\ Fax: (++39) 0412349176 \\ e-mail: billio@unive.it
}

his Working Paper is published under the auspices of the Department of Economics of the Ca' Foscari University of Venice. Opinions expressed herein are those of the authors and not those of the Department. The Working Paper series is designed to divulge preliminary or incomplete work, circulated to favour discussion and comments. Citation of this paper should consider its provisional character.

$\begin{aligned} \begin{array}{r}\text { The Working Paper Series } \\ \text { is availble only on line }\end{array} & \begin{array}{l}\text { Department of Economics } \\ \text { Ca' Foscari University of Venice }\end{array} \\ \text { (www.dse.unive.it/pubblicazioni) } & \text { Cannaregio 873, Fondamenta San Giobbe } \\ \text { For editorial correspondence, please contact: } & \text { 30121 Venice Italy } \\ \text { wp.dse@unive.it } & \text { Fax: ++39 041 2349210 }\end{aligned}$




\section{Introduction}

In the last few years, the empirical and theoretical analysis concerning multivariate GARCH models attracted a growing interest for two main reasons: the availability of more and more powerful computers that enabled the estimation of complex models with an elevate number of parameters and the introduction of a new class of models: the Dynamic Conditional Correlation multivariate GARCH (DCC) by Engle (2002). Several generalizations of the Engle's model as been proposed (among others Cappiello et al. 2006, and Franses and Hafner, 2003, Billio et al., 2006) and some theoretical studies have also been developed (McAleer et al., 2006). These papers focused both on the developments of new parameterizations and on their use in empirical applications, demonstrating an high capability to adapt to practical problems.

In this paper we introduce a new DCC-type model that generalized the Flexible DCC of Billio et al. (2006), FDCC. We start from the empirical evidence that in asset allocation problems we need flexible and feasible models to construct optimal portfolios. Asset managers generally invest by differentiating their portfolio by area, branches or sectors and type of instruments. This fact suggests to develop the block structure in the parameters of the FDCC model, thus allowing for constant dynamics only among block of assets belonging to the same category. Our model generalizes the FDCC structure by allowing for possible interactions in the correlation dynamics among classes of assets.

In Section 2 we review current DCC type models and in section 3 we introduce the Quadratic FDCC model also providing conditions for positive definiteness and stationarity. Section 3 reports an empirical example based on the sectorial indices of the Italian Stock Market (MIBTEL). Section 4 concludes.

\section{Modeling Dynamic Conditional Correlations}

The starting point for the analysis of dynamic correlation models is the Constant Condition Correlation model of Bollerslev (1991). In his paper, Bollerslev assumed that the variance-covariance matrix of $\varepsilon_{t}$ (a $K$-dimension set of asset returns) could be factorized as follows:

$$
H_{t}=D_{t} R D_{t}
$$

where $R$ is a correlation matrix and $D_{t}$ is a diagonal matrix of conditional volatilities. For the sake of exposition we assume that the mean is not relevant. For each series a GARCH-type model could be fitted for estimating the conditional variance without any constraint on a common structure. Each conditional variance could be modelled with a standard GARCH model or with more advanced parameterisations such as GARCH models with asymmetry effects as in Glosten et al. (1993) and in Caporin and McAleer (2006), or EGARCH representations as in Nelson (1991). 
The representation with constant conditional correlations allows for a twostep estimation procedure: at first, we estimate the conditional variances, which can then be filtered out by premultiplying $\varepsilon_{t}$ by $D_{t}^{-1}$; then, the correlation matrix can be estimated. Furthermore, we can estimate the correlation with the simple sample estimator

$$
R=\frac{D_{t}^{-1} \varepsilon_{t} \varepsilon_{t}^{\prime} D_{t}^{-1}}{T}
$$

Unfortunately, the assumption of constant correlations is really questionable. In fact, it is well-known that correlations are not stable over long periods. Engle (2002) introduced a limited dynamics into the correlations in order to overcome this limitation. Engle restated the decomposition (1) as:

$$
\begin{gathered}
H_{t}=D_{t} R_{t} D_{t} \\
R_{t}=\left(Q_{t}^{*}\right)^{-1} Q_{t}\left(Q_{t}^{*}\right)^{-1}
\end{gathered}
$$

where he assumed that the time-dependent correlation has a quadratic structure (which was added to ensure that we have, at the end, a correlation matrix). Furthermore, $Q_{t}$ has the following expression

$$
Q_{t}=[1-\alpha(1)-\beta(1)] \Gamma+\alpha(L) \boldsymbol{\eta}_{t-1} \boldsymbol{\eta}_{t-1}^{\prime}+\beta(L) Q_{t-1}
$$

where: $\boldsymbol{\eta}_{t}=D_{t}^{-1} \varepsilon_{t}$; the polynomial parameters are defined as $\alpha(L)=\sum_{i=1}^{\bar{q}} \alpha_{i} L^{i}$, $\beta(L)=\sum_{i=1}^{\bar{p}} \beta_{i} L^{i}$ and must satisfy $\alpha(1)+\beta(1)<1$ in order to rule out explosive patterns and $\Gamma=T^{-1} \sum_{i=1}^{T} \boldsymbol{\eta}_{t} \boldsymbol{\eta}_{t}^{\prime}$ is the unconditional (sample) correlation matrix. The dynamics is thus very similar to a GARCH-type equation. Furthermore, the unconditional correlations are equal to the sample correlations (i.e. unconditionally $Q=[1-\alpha(1)-\beta(1)] \Gamma+\alpha(L) Q+\beta(L) Q \Longrightarrow Q=R=\Gamma$ ); we will refer to this result as the "correlation targeting" property. Finally, $Q_{t}^{*}=\operatorname{diag}\left(\sqrt{q_{11, t}}, \sqrt{q_{22, t}}, \ldots \sqrt{q_{n n, t}}\right)$.

A very similar approach is included in the paper of Tse and Tsui (2002), the only difference is in the term $\boldsymbol{\eta}_{t-1} \boldsymbol{\eta}_{t-1}^{\prime}$ which is substituted by a short term correlation estimate $m^{-1} \sum_{j=1}^{m} \boldsymbol{\eta}_{t-j} \boldsymbol{\eta}_{t-j}^{\prime}$ with $m \geq K$.

This model is clearly parsimonious since it requires just two parameters to introduce dynamics into correlations. However, it implies several strong restrictions: first, there is no interdependence among variances, among correlations and between variances and correlations; second, the dynamics is constant over all correlations.

We can solve the first point only moving from the DCC model to standard multivariate GARCH models like the Vech or the BEKK of Engle and Kroner (1995). These two models allow for interdependence among variances and covariances and thus they implicitly assume dynamic correlations, even if their focus being on dynamic covariances. Unfortunately, BEKK and Vech models are useless in systems with more that 4 or 5 variables since they have 
serious optimization problems leading to unstable and inconsistent parameter estimates. Differently, the empirical interest is in models with many assets, possibly more than 100 . One solution is then to split the problem, estimating conditional variances on a univariate basis and focusing in a second step on the correlations. Clearly, the use of a two-step approach provides important computational advantages, but it excludes any direct interaction among covariances. The introduction of lagged cross-sectional dependence between the variances could follow standard models, as the VARMA-GARCH of Ling and McAleer (2003). We will not directly address this issue since the focus of the paper is on correlation modelling. Anyway, we underline that the correct specification of the variance dynamics is fundamental. In fact, the dynamic evolution of the correlation could be influenced by a possible misspecification of the variance equations. We thus face a trade-off between the use of an advanced and possibly multivariate specification of the variance evolution and the model feasibility.

Within the dynamic correlation literature, the most common approach considers univariate specification of the variances, possibly including asymmetric terms following the GJR model of Glosten et al. (1993). In the empirical application we will follow this strategy.

The second limitation, given by the constancy of the dynamics over all the correlations, has been already addressed in the econometric literature. The DCC model was generalized by Engle (2002), who suggested the following Generalized DCC trying to solve the constraint of equal dynamics for all correlations

$$
Q_{t}=\left[i i^{\prime}-A-B\right] \circ \Gamma+A \circ \boldsymbol{\eta}_{t-1} \boldsymbol{\eta}_{t-1}^{\prime}+B \circ Q_{t-1}
$$

where $\circ$ is the Hadamard product (elementwise matrix multiplication), $A$ and $B$ are square matrices and positive definiteness is guaranteed by their positive definiteness (see Ding and Engle, 2001). This model solved one of the drawback of the original DCC but, unfortunately, the number of parameters greatly increases and makes the model empirically unattractive.

Additional extensions shortly appeared in the literature:

i) Cappiello et al. (2006) provide a different extension of the DCC model by introducing asymmetry in the correlation dynamics and translating the model into a quadratic form

$Q_{t}=\left(\Gamma-A^{\prime} \Gamma A-B^{\prime} \Gamma B-G^{\prime} \bar{F} G\right)+A^{\prime} \boldsymbol{\eta}_{t-1} \boldsymbol{\eta}_{t-1}^{\prime} A+B^{\prime} Q_{t-1} B+G^{\prime} \xi_{t-1} \xi_{t-1}^{\prime} G$

where $\xi_{t}=I\left(\boldsymbol{\xi}_{t}<0\right) \circ \boldsymbol{\xi}_{t}, A, B, G$ are diagonal parameter matrices, $\Gamma$ is again the sample covariance matrix of the standardized residuals and $\bar{F}$ is the sample covariance matrix of $\xi_{t}$; this model adds flexibility to the previous one, however the number of parameters increases with system dimension and the positive definiteness is obtained by constraining the matrix $\bar{Q}-A^{\prime} \bar{Q} A-$ $B^{\prime} \bar{Q} B-G^{\prime} \bar{F} G$ to be positive definite, which is quite a complex task; 
ii) Franses and Hafner (2003) suggested another Generalized DCC model

$$
Q_{t}=\left[1-\sum_{i=1}^{n} \alpha_{i}-\beta\right] \Gamma+\alpha \alpha^{\prime} \circ \boldsymbol{\eta}_{t-1} \boldsymbol{\eta}_{t-1}^{\prime}+\beta Q_{t-1}
$$

with $\alpha$ being a vector of dimension $n$. Here the positive definiteness is guaranteed without constraints but the correlation targeting property is no more valid;

iii) McAleer et al. (2006) generalize the model providing a representation in which all the dynamic correlations can have a different dynamic pattern; their approach is particularly useful from a theoretical point of view, since it provides regularity conditions for the moments and the asymptotic properties of the quasi maximum likelihood estimator applied to dynamic correlation models (and the DCC models of Engle (2002) are special cases);

iv) Billio et al. (2006) suggest two special cases of the Generalized DCC of Engle (2002) and Franses and Hafner (2003), by requiring that the parameter matrices or parameter vectors is partitioned. The intuition behind this choice is that the dynamics cannot be common for all correlations but a too generalized parameterization is not feasible; therefore, they suggest to group variables in coherent sets mirroring the empirical needs of sectorial or geographical asset allocation (i.e. stocks from Europe and Asia or belonging to the Energy and Financial sectors); in formula (4), they required that

$$
A=\left[\begin{array}{cccc}
\alpha_{i, 11} i\left(m_{1}\right) i\left(m_{1}\right)^{\prime} & \alpha_{i, 12} i\left(m_{1}\right) i\left(m_{2}\right)^{\prime} & \cdots & \alpha_{i, w 1} i\left(m_{1}\right) i\left(m_{w}\right)^{\prime} \\
\alpha_{i, 12} i\left(m_{2}\right) i\left(m_{1}\right)^{\prime} & \alpha_{i, 22} i\left(m_{2}\right) i\left(m_{2}\right)^{\prime} & & \alpha_{i, w 2} i\left(m_{2}\right) i\left(m_{w}\right)^{\prime} \\
\vdots & & \ddots & \vdots \\
\alpha_{i, w 1} i\left(m_{w}\right) i\left(m_{1}\right)^{\prime} & \alpha_{i, w 2} i\left(m_{w}\right) i\left(m_{2}\right)^{\prime} & \cdots & \alpha_{i, w w} i\left(m_{w}\right) i\left(m_{w}\right)^{\prime}
\end{array}\right]
$$

where $m_{1}, m_{2}, \ldots m_{w}$ are the number of assets in each group (similarly for $B$ ); in that case the correlation matrix is positive definite if so is the matrix $\left[i i^{\prime}-A-B\right] \mathrm{o}$ $\Gamma$; the constraints are heavy but feasible if off-diagonal blocks are filled up with zeros (i.e. only $\alpha_{i, j j} \neq 0$ ); they named this particular DCC the Block-Diagonal DCC (BDDCC) model. To solve this further limitation they generalize the model of Franses and Hafner (2003) adding the constant

$$
Q_{t}=c c^{\prime} \circ \Gamma+\alpha \alpha^{\prime} \circ \boldsymbol{\eta}_{t-1} \boldsymbol{\eta}_{t-1}^{\prime}+\beta \beta^{\prime} \circ Q_{t-1}
$$

and requiring the parameter vectors to be partitioned vectors, as $\alpha=\left\{\alpha_{1}, \alpha_{1}, \alpha_{1}, \alpha_{1}, \alpha_{2}, \alpha_{2}, \alpha_{3}, \alpha_{3}, \alpha_{3},\right\}$; in that case they gain the positive definiteness but loose, in general, the correlation targeting property, as in the Franses and Hafner (2003) model. Differently from their approach, Billio et al. (2006) can impose positive definiteness with the following constraints $\alpha_{i} \alpha_{j}+\beta_{i} \beta_{j}+c_{i} c_{j}=1$ for $i, j=1 \ldots n$; they labeled this model the Flexible DCC; in both cases the parameters of the GARCH part (not the constant) must satisfy a "stationarity" constraint $\alpha_{i} \alpha_{j}+\beta_{i} \beta_{j}<1$. McAleer (2005) and Bauwens et al. (2006) provide an extensive survey on multivariate correlation models. 


\section{The Quadratic Flexible DCC Model}

In this paper we introduce a new DCC-type mode which generalize the Flexible DCC: the Quadratic Flexible DCC can be also considered a special case of the Asymmetric DCC of Engle, Cappiello and Sheppard (2006). We suggest the following parameterization of $Q_{t}$ :

$$
Q_{t}=C^{\prime} \Gamma C+A^{\prime} \boldsymbol{\eta}_{t-1} \boldsymbol{\eta}_{t-1}^{\prime} A+B^{\prime} Q_{t-1} B
$$

where $A, B$ and $C$ are symmetric matrices. This model nests the FDCC which correspond to a Quadratic FDCC with diagonal partitioned parameter matrices. As the FDCC this model generally looses the correlation targeting property which can however be imposed with a set of restrictions. The quadratic structure of the model guarantees the positive definiteness of $Q_{t}$, given a suitable starting point. A comment on parameter constraint is worthwhile: in standard DCC the $\alpha$ and $\beta$ parameters must satisfy a constraint $(\alpha+\beta<1)$ that rules out explosive correlation patterns; the QFDCC model requires a similar constraint but it must be imposed on the eigenvalues of $A+B$. In fact, the QFDCC can be thought as a particular BEKK model once the variance effect has been filtered out. Then, following Engle and Kroner (1995) we can recast the QFDCC in a companion Vech-type form and use their Proposition 2.7. Consequently, the QFDCC model provides stationary correlations if: i) $C^{\prime} \Gamma C$ is positive definite; ii) the eigenvalues of $A+B$ are in modulus less than 1 .

In the QFDCC model we can adapt block structures to the parameter matrices as in (7). Finally, by removing the assumption of diagonal parameter matrices, as in the Asymmetric DCC, the QFDCC model allows for interdependence among correlations. Clearly, a completely unrestricted model is unfeasible from an estimation point of view. For this reason, we suggest several special cases: with diagonal parameter matrices as in the A-DCC (5); with partitioned diagonal matrices, similarly to the FDCC (8); finally, block-partitioned representations could be adopted as in BDDCC (7). We present a particular example for this last case.

Assume that we are considering a system with $K=5$ assets grouped into two sets of $n_{1}=3$ and $n_{2}=2$ assets, respectively. Also assume the following structures for the parameter matrices

$$
\begin{aligned}
A= & {\left[\begin{array}{ccccc}
0 & 0 & 0 & a_{2} & a_{2} \\
0 & 0 & 0 & a_{2} & a_{2} \\
0 & 0 & 0 & a_{2} & a_{2} \\
a_{2} & a_{2} & a_{2} & a_{1} & 0 \\
a_{2} & a_{2} & a_{2} & 0 & a_{1}
\end{array}\right] \quad B=\left[\begin{array}{ccccc}
0 & 0 & 0 & b_{2} & b_{2} \\
0 & 0 & 0 & b_{2} & b_{2} \\
0 & 0 & 0 & b_{2} & b_{2} \\
b_{2} & b_{2} & b_{2} & b_{1} & 0 \\
b_{2} & b_{2} & b_{2} & 0 & b_{1}
\end{array}\right]{ } } \\
C= & {\left[\begin{array}{ccccc}
1 & 0 & 0 & c_{2} & c_{2} \\
0 & 1 & 0 & c_{2} & c_{2} \\
0 & 0 & 1 & c_{2} & c_{2} \\
c_{2} & c_{2} & c_{2} & c_{1} & 0 \\
c_{2} & c_{2} & c_{2} & 0 & c_{1}
\end{array}\right] }
\end{aligned}
$$


Then, by substitution on (9) we can verify that: i) the interdependence between correlations can be handled with a very limited number of parameters; i) the QFDCC model allows the combination of constant correlations for some assets and dynamic correlations for others (simply imposing the restrictions $c_{2}=b_{2}=$ $\left.a_{2}=0\right)$. Furthermore, we can estimated a generalized model and run some likelihood ratio test for nested models. Finally, the QFDCC can be generalized adding asymmetry terms following the strategy proposed by Cappiello et al. (2006).

\subsection{Estimation and Testing}

Maximum likelihood is the standard tool for the estimation of dynamic conditional correlation models presented in this work. Following Engle (2002), let $\theta_{1}$ be the parameter set of the univariate GARCH models and $\theta_{2}$ the parameter set of the dynamic correlation structure. We can represent the likelihood of the model as follows:

$$
\log L\left(\theta_{1}, \theta_{2} \mid X_{t}\right)=-\frac{1}{2} \sum_{t=1}^{T}\left[k \log (2 \pi)+\log \left(\left|H_{t}\right|\right)+\varepsilon_{t} H_{t}^{-1} \varepsilon_{t}^{\prime}\right]
$$

Further, exploiting the relation (3) we can write:

$\log L\left(\theta_{1}, \theta_{2} \mid X_{t}\right)=-\frac{1}{2} \sum_{t=1}^{T}\left[k \log (2 \pi)+\log \left(R_{t}\right)+2 \log \left(\left|D_{t}\right|\right)+\varepsilon_{t} D_{t}^{-1} R_{t}^{-1} D_{t}^{-1} \varepsilon_{t}^{\prime}\right]$

Engle suggested a first estimation stage where the correlation matrix has to be replaced by an identity matrix

$$
\log L\left(\theta_{1} \mid X_{t}\right)=-\frac{1}{2} \sum_{t=1}^{T}\left[k \log (2 \pi)+\log \left(I_{n}\right)+2 \log \left(\left|D_{t}\right|\right)+\varepsilon_{t} D_{t}^{-1} I_{n}^{-1} D_{t}^{-1} \varepsilon_{t}^{\prime}\right]
$$

This step is equivalent to univariate estimation of GARCH models. In a second step, conditionally on the parameters estimated in the first step, we have the following log-likelihood

$$
\log L\left(\theta_{2} \mid \hat{\theta}_{1}, X_{t}\right)=-\frac{1}{2} \sum_{t=1}^{T}\left[k \log (2 \pi)+\log \left(R_{t}\right)+2 \log \left(\left|D_{t}\right|\right)+\boldsymbol{\eta}_{t} R_{t}^{-1} \boldsymbol{\eta}_{t}^{\prime}\right]
$$

where $\boldsymbol{\eta}_{t}=D_{t}^{-1} \varepsilon_{t}$ are the first stage standardized residuals.

According to the results of Comte and Lieberman (2003), Ling and McAleer (2003) and McAleer et al. (2006), the maximum likelihood estimators are consistent and asymptotically normally distributed.

Given the relations between Engle's DCC, the Flexible DCC and the Quadratic FDCC model we can apply several likelihood tests for parameter restrictions. The LR tests have an asymptotic chi-square distribution under the assumptions and regularity conditions stated in Comte and Lieberman (2003), Ling 
and McAleer (2003) and McAleer et al. (2006). We stress that our working hypothesis will never be an unrestricted QFDCC, which is not feasible, but instead we consider the Block QFDCC (with block partitioned parameter matrices). In order to evidence all the possible bivariate model comparison we consider the two-block example. In that case, $A$ is defined as follows

$$
A=\left[\begin{array}{cc}
a_{1} i_{n_{1}} i_{n_{1}}^{\prime} & a_{12} i_{n_{1}} i_{n_{2}}^{\prime} \\
a_{12} i_{n_{2}} i_{n_{1}}^{\prime} & a_{2} i_{n_{2}} i_{n_{2}}^{\prime}
\end{array}\right]=\left[\begin{array}{cc}
A_{1} & A_{12}^{\prime} \\
A_{12} & A_{2}
\end{array}\right],
$$

and similarly $B$ and $C$. We can then test the block structured benchmark model against a set of alternative parameterizations:

i) Block QFDCC against Block Diagonal QFDCC: this is obtained by restricting to 0 all off-block diagonal coefficients, $a_{12}=b_{12}=c_{12}=0$;

ii) the benchmark model with respect to a structure with diagonal blocks restricted to be diagonal: in that case, we assume that the correlations belonging to a given diagonal block have no feedback effects (that is, they are simply characterized by the same dynamic behavior): in that case we impose $A_{1}=$ $a_{1} I_{n_{1}}$, and similar representations are used for $A_{2}, B_{1}, B_{2}, C_{1}, C_{2}$;

iii) Block QFDCC against a Diagonal QFDCC: this is the restriction that implies an FDCC model where there is no interdependence across correlations; this is equivalent to merging restrictions i) and ii);

iv) our benchmark model can be also compared to Engle's DCC model; this is equivalent to the following set of restrictions: $A_{1}=A_{2}=a I_{n_{1}+n_{2}}$, $B_{1}=B_{2}=b I_{n_{1}+n_{2}}$, (similar to i) and ii) but excluding the constant term $C$ ), $c_{1}=c_{2}=\sqrt{1-a^{2}-b^{2}}$ (given that we are using a quadratic form), and $c_{12}=0$;

v) a CCC model, that is $A=B=0$ and $C=I_{n}$.

Additional restrictions could be considered for testing mixed models such as the one proposed in (10). In addition, the information criteria can be used to compare the QFDCC with non-nested models like the Franses and Hafner DCC.

\section{Portfolio Risk Evaluation with DCC-type mod- els}

Dynamic correlation models may provide useful insights in several financial applications including asset allocation within a Markowitz approach, forecast evaluation analysis and portfolio risk evaluation. In this paper we focus on this last case using a set of stock market indices. In details, we consider the main Italian stock market index, the Mibtel, and its sectorial decomposition which we report in Table 1.

[INSERT Figure 1 - Mibtel sectorial decomposition]

The data were downloaded from Datastream and cover the range January 1991 to September 2003 at the daily frequency. The index has two levels of 
disaggregation. In the first, the index is decomposed in three main groups Industrial, Service and Finance. These three indices are further disaggregated into a group of 20 sub-sectors. The whole sample consists of about 3400 observations. Following a standard practice, we fitted univariate GARCH models with asymmetry following Glosten et al. (1997) on the log-returns of the sub-sector indices. Table 2 reports the estimated parameters and the quasi maximum likelihood standard errors. All sub-sectors conditional variances show a relevant asymmetric effect and only three reports a GARCH coefficient lower than 0.7. Given the comments reported in section 2 and the focus on correlation dynamics, we did not consider further GARCH specifications.

\section{[INSERT Table 1 - Univariate GARCH estimates]}

Following the approach of Engle (2002), after the estimate of the conditional variance models, we compute the standardized residuals. On the resulting series we fit then dynamic correlation models. As benchmark model we computed the sample (unconditional) correlation matrix on the standardized residuals. This estimate is equivalent to a Constant Conditional Correlation model. In this case the correlation model likelihood is equal to -9842.368 .

We also computed the unconditional correlations (again on the standardized residuals) using a rolling window of 250 observations. Some patterns are reported in Figures 1 and 2. This graphical analysis evidences that correlations are not stable over time and, more interestingly, that correlations show similar patterns between sub-sectors groups.

[Insert Figures 2 and 3- rolling correlations]

This graphical analysis suggests that the QFDCC should be considered as a valid alternative to the excessively restricted DCC and CCC representations. Tables 2 and 3 reports parameters estimates for the whole sample for DCC and for the diagonal QFDCC models. The log-likelihoods suggest that the QFDCC model should be preferred to the DCC (it provides a small but significant increase in the likelihood). However, the improvements achieved with the QFDCC (and only with a diagonal representation) are very relevant, suggesting that even small increases in model flexibility may provide valid and preferred representations. Standard likelihood ratio tests strongly support these findings.

[INSERT Table 2and 3 parameter estimates of DCC and QFDCC]

Our final purpose is to compare the performances of CCC, DCC and QFDCC in evaluating portfolio risk. For this reason, we focus on the last two years of our sample and we estimate the various models in a rolling window of 250 observations and a step of 10 observations. This correspond roughly to a portfolio allocation and evaluation which is updated every two weeks. In order to get directly comparable portfolios in term of returns and avoid any discussion on 
the estimation of mean expected returns, we consider equally weighted portfolios (i.e. the $5 \%$ of the global portfolio is invested in each of the 20 sub-sectors indices of the Mibtel). The various portfolios are then equivalent in terms of returns but not in their exposition to market risk which is influenced by the second order moments. We compare the correlation models using the Value-at-Risk measure with a backtesting procedure.

The VaR is the quantile of portfolio returns $\left(r_{t}\right)$ satisfying

$$
\int_{-\infty}^{V a R(t, \alpha)} r_{t} f\left(r_{t}\right) d r_{t}=\alpha .
$$

The backtesting analysis considers a comparison over the last 250 days and focuses mainly on exceptions: i.e. the number of cases in which the portfolio returns underperform the VaR measure. In that case, we also computed the RiskMetrics model (RM) (JP Morgan, 1996), which is the alternative benchmark model extremely popular in the literature. The RM model considers that variances and correlations follow an exponentially weighted moving average. Define the returns on a sub-sector index as $r_{i, t}$, denote the variance-covariance matrix of the 20 indices by $\Sigma_{t}$, and let $\omega$ be the row-vector of portfolio weights (each element of the vector is equal to $1 / 20$ (we are considering an equally weighted portfolio). Then, time varying portfolio returns are:

$$
r_{t}=\omega\left[\begin{array}{c}
r_{1, t} \\
r_{2, t} \\
\vdots \\
r_{20, t}
\end{array}\right]
$$

while portfolio variances are:

$$
\sigma_{t}^{2}=\omega \Sigma_{t} \omega^{\prime}
$$

Note that portfolio weights are repositioned at the equally weighted level every 10 days while the variance-covariance matrix is estimated with a CCC (i.e. with constant correlations), a DCC, a QFDCC and finally with the RiskMetrics model. In this last case, we estimate the elements of $\Sigma_{t}$ using the recursive formula

$$
\sigma_{i, t} \sigma_{j, t}=\lambda \sigma_{i, t} \sigma_{j, t}+(1-\lambda) r_{i, t} r_{j, t} \quad i, j=1 \ldots 20
$$

and $\lambda$ set to 0.97 . Table 4 reports the exceptions realized by the three correlation models and by the RM.

[Table 4 - exceptions]

Among the various models, only the QFDCC and the RM models provide an exception number very close to the theoretical value. Differently, CCC and DCC report the same result, which is too conservative. They provide a lower exception number, which indicates that the portfolio variances provided by these 
two approaches are larger than the one provided by RM and QFDCC. This fact is not negative if our final purpose is to adequately cover market risk exposure. However, a more conservative VaR methodology necessarily implies a larger opportunity cost: larger $\mathrm{VaR}$ is equivalent to a larger amount of immobilized resources.

A comparison between correlation models cannot be based only on counting the exceptions, but further metric must be considered. The literature focuses on two standard approaches: testing for VaR model failures, following Kupiec (1995); comparing models with loss functions, following Lopez (1998). In this paper we combine these standard techniques with some additional measures proposed in Caporin (2003). Kupiec (1995) suggests two tests for the evaluation of VaR measures: the Proportion of Failure test and the Test Until First Failure. Both tests are based on the assumption that exceptions follow a binomial distribution and are asymptotically distributed as a chi-square variable with one degree of freedom. Both tests are used to verify the null hypothesis that VaR measures are correctly specified. Table 7 reports the tests for the various correlation models.

[Table 5 - tests for VaR]

Even in this case, the CCC and DCC models provide the worst results: larger test statistics, closer to the rejection area; rejection of the null hypothesis in the $10 \%$ VaR case; finally, the tests allow to infer that the two models provide exactly the same exceptions. Differently, RM and QFDCC provide comparable test statistics.

Unfortunately, it is well-know that these tests have limited power in distinguishing among various models for VaR, see among other Lopez (1998). Loss functions represents an alternative approach that can be used to compare VaR models. These loss functions can be appropriately designed in order to overcome the tests limitations. Lopez (1998) suggests a loss function based on regulatory needs:

$$
L f_{L}=\sum_{t=1}^{T}\left[1+\left(r_{t}-V a R_{t}\right)^{2}\right] I_{t}\left(r_{t}<V a R_{t}\right)
$$

where $I_{t}$ is an indicator function that selects exceptions. This loss function penalizes the VaR models that provide largest losses at the exceptions. However, a bank would prefer a VaR model that: (i) satisfy Basel Accord requirements, (ii) reduces losses at the exceptions, (iii) and also reduces the opportunity cost of VaR (the VaR also measures the amount of money that a financial institutions must immobilize to cover market risk exposure). A VaR model that satisfies points i) and ii) and that provides lower bounds than the other is clearly preferred, since it translates in lower immobilization of liquidity resources).

Lopez loss function focus only on point (i); additional metrics are therefore needed. Caporin (2003) introduces alternative loss functions that can be used to compare models in terms of (ii) and (iii). The loss functions focus on the distances between the VaR bounds and the realized portfolio returns. Therefore 
they can be used on the whole return path and not only on the exceptions. We report here two loss functions, that can be thought as first and second order losses

$$
\begin{gathered}
L f_{F}=\sum_{t=1}^{T} \frac{\left|r_{t}\right|-\left|V a R_{t}\right|}{\left|V a R_{t}\right|} \\
L f_{S}=\sum_{t=1}^{T} \frac{\left(\left|r_{t}\right|-\left|V a R_{t}\right|\right)^{2}}{\left|V a R_{t}\right|} \\
L f_{C}=L f_{F}+L f_{S} .
\end{gathered}
$$

[Table 6 - loss functions]

If the attention is given only to the exceptions, then the Lopez loss function should be used. In that case, the RM model provide the best result at the $1 \%$ level, while the optimal model is the DCC at the $5 \%$ and $10 \%$ Value-at-Risk level; at the opposite, the QFDCC is the worst case. This in turn implies that, at the exceptions, the QFDCC provide a lower portfolio variance compared to the other models; it is less conservative than the other models and this give rise to larger losses. If we move from the exception cases to the whole path of the portfolio variances, we should consider the alternative loss functions previously introduced. These loss functions have been calculated over the full back-testing period and not only over the exceptions. In that case, we note that the result is completely reversed: the QFDCC model is the optimal choice at $1 \%$ and $5 \%$ cases while the RM is the best model for a $10 \%$ VaR. Collecting these results we can state the following: regulators should prefer a Lopez loss function approach for comparing VaR models while financial institutions should push for the use of different loss functions. In fact, a more flexible approach which satisfies the Basel Accord Requirements and provides lower VaR bounds could reduces the opportunity cost of immobilizing resources.

Finally, we consider a further analysis on VaR bounds using correlations among them and awe also compare the VaR levels at various quantiles. The purpose of this additional analysis is to verify if the correlation models we considered provide VaR bounds close one to the other. A high correlation between two sequences of exceptions suggest that the two models detect the very same VaR exceptions. Similarly, a high correlation between VaR bounds evidences that the proposed models provide similar portfolio variance dynamics. Table 7 reports the correlations among VaR bounds and among the sequences of exceptions at $1 \%, 5 \%$ and $10 \%$ VaR, respectively.

[Table 7 correlations]

It clearly emerges that: $\mathrm{CCC}$ and DCC models provide the same exceptions as we previously noted; QFDCC model is close to the DCC one; and, finally, that the RM model is far from the DCC-type models, in particular at $1 \%$ and $5 \%$ VaR levels. 
Finally, table 8 reports the VaR exceptions at various quantiles, from $1 \%$ to $30 \%$, together with the theoretical exception values. We can note that all the models are much more conservative increasing the quantile probability.

[Table 8 quantiles and exceptions]

Summarizing our findings, we can state that the QFDCC model provides a significant increase in the log-likelihood compared to standard alterative correlation models. Furthermore, if the comparison is based on a portfolio risk evaluation framework, the QFDCC model produces exceptions closer to the theoretical values. Finally, using loss functions we verify that the CCC and DCC models generally provide wider VaR bounds that satisfy Basel Accord requirements but also imply a higher opportunity cost.

\section{Conclusions}

This paper introduces a new dynamic conditional correlation model, the Quadratic Flexible DCC, that generalizes the DCC model of Engle (2002) and the FDCC model of Billio et al. (2006). The model allows for interaction among correlations with a quadratic structure similar to the one included in the BEKKGARCH model of Engle and Kroner (1995). Furthermore, differently from the DCC, a constant is included to guarantee more flexibility. Finally, the parameters are imposed to be constant across clusters of assets that can be defined a priori.

Following this approach, the parameter number greatly reduces and parameter matrices become partitioned matrices. The use of block-structure parameter matrices provides relevant advantages and a limited increase in model complexity. The proposed approach could be used in most multivariate GARCH models, including the BEKK of Engle and Kroner (1995), and in most of the parameterizations described in McAleer (2005) and Bauwens et al. (2006). Furthermore, the use of block-structures and quadratic forms could also be considered within a multivariate stochastic volatility framework extending the models presented by Asai et al. (2006).

The QFDCC model is designed to be used in empirical finance applications involving asset management and risk evaluation. This paper provided an empirical analysis in this second area, considering the VaR computation with different approaches, including CCC, DCC, QFDCC and the RiskMetrics models. In that particular case, the QFDCC model provides the best results on most cases providing a number of exceptions in line with Basel Accord requirements and a narrower VaR bounds. 


\section{References}

[1] Asai, M., M. McAleer and J. Yu, 2006, Multivariate stochastic volatility: a review, Econometric Reviews, 25, 145-175

[2] Bauwens L., S. Laurent and J.K.V. Rombouts, 2006, Multivariate GARCH models: a survey, Journal of Applied Econometrics, 21, 79-109

[3] Billio, M., M. Caporin and M. Gobbo, 2006, Flexible dynamic conditional correlation multivariate GARCH for asset allocation, Applied Financial Economics Letters, 2, 123-130

[4] Bollerslev T., 1990, Modelling the coherence in short-run nominal exchangerates: a multivariate generalized $\mathrm{ARCH}$ approach, Review of Economic Studies, 72, 498-505

[5] Caporin, M., 2003, Comparing Value-at-Risk measures in presence of long memory conditional variances, GRETA Working Paper, proceedings of the ASSET 2002 Conference, Cyprus, November 2002.

[6] Caporin, M. and M. McAleer, 2006, Dynamic Asymmetric GARCH, Journal of Financial Econometrics, 4(3), 385-412

[7] Cappiello L., R.F. Engle and K. Sheppard, 2006, Asymmetric dynamics in the correlations of global equity and bond returns, Journal of Financial Econometrics, 25, 537-572

[8] Comte, F. and O. Lieberman, 2003, Asymptotic theory for multivariate GARCH processes, Journal of Multivariate Analysis, 84(1), 61-84

[9] Ding, Z. and R.F. Engle, 2001, Large scale conditional covariance matrix modeling estimation and testing, Academia Sinica Papers, 29, 157-184

[10] Engle R.F. and K.F. Kroner, 1995, Multivariate simultaneous generalized ARCH, Econometric Theory, 11, 122-150

[11] Engle, R.F., 2002, Dynamic conditional correlation: a simple class of multivariate generalized autoregressive conditional heteroskedasticity models, Journal of Business and Economic Statistics, 20, 339-350.

[12] Engle R.F. and K. Sheppard, 2001, Theoretical and empirical properties of dynamic conditional correlation multivariate GARCH, University of California, San Diego, Discussion paper 2001-15, NBER Working Paper 8554

[13] Franses, P.H. and C.M. Hafner, 2003, A Generalised Dynamic Conditional Correlation Model for Many Asset Returns, Econometric Institute Report EI 2003-18, Erasmus University Rotterdam

[14] Glosten, L.R., R. Jagannathan and D.E. Runkle, 1993, On the relation between the expected value and the volatility of the nominal excess return on stocks, The Journal of Finance, 48-5, 1779-1801 
[15] Ling, S. and M. McAleer, 2003, Asymptotic theory for a Vector ArmaGarch model, Econometric Theory, 19-2, 280-310

[16] JP Morgan, 1996, RiskMetrics Technical Document, 4th edition, New York

[17] Kupiec, H., 1995, Techniques for verifying the accuracy of risk measurement models, The journal of Derivatives, 73-84

[18] Lopez A.J., 1999, Methods for evaluating Value-at-Risk estimates, Federal Reserve Bank of San Francisco Economic Review, 2

[19] McAleer, M., 2005, Automated inference and learning in modeling financial volatility, Econometric Theory, 21, 232-261.

[20] McAleer, M., F. Chan, S. Hoti and O. Lieberman, 2006, Generalised Autoregressive Conditional Correlation, preprint

[21] Nelson, D.B., 1991, Conditional heteroskedasticity in asset retursn: a new approach, Econometrica, 59, 347-370

[22] Tse Y.K. and A.K.C. Tsui, 2002, A multivariate Generalises Autoregressive Conditional Heteroscedasticity model with time-varying correlations, Journal of Business and Economic Statistics, 20 (3), 351-362 


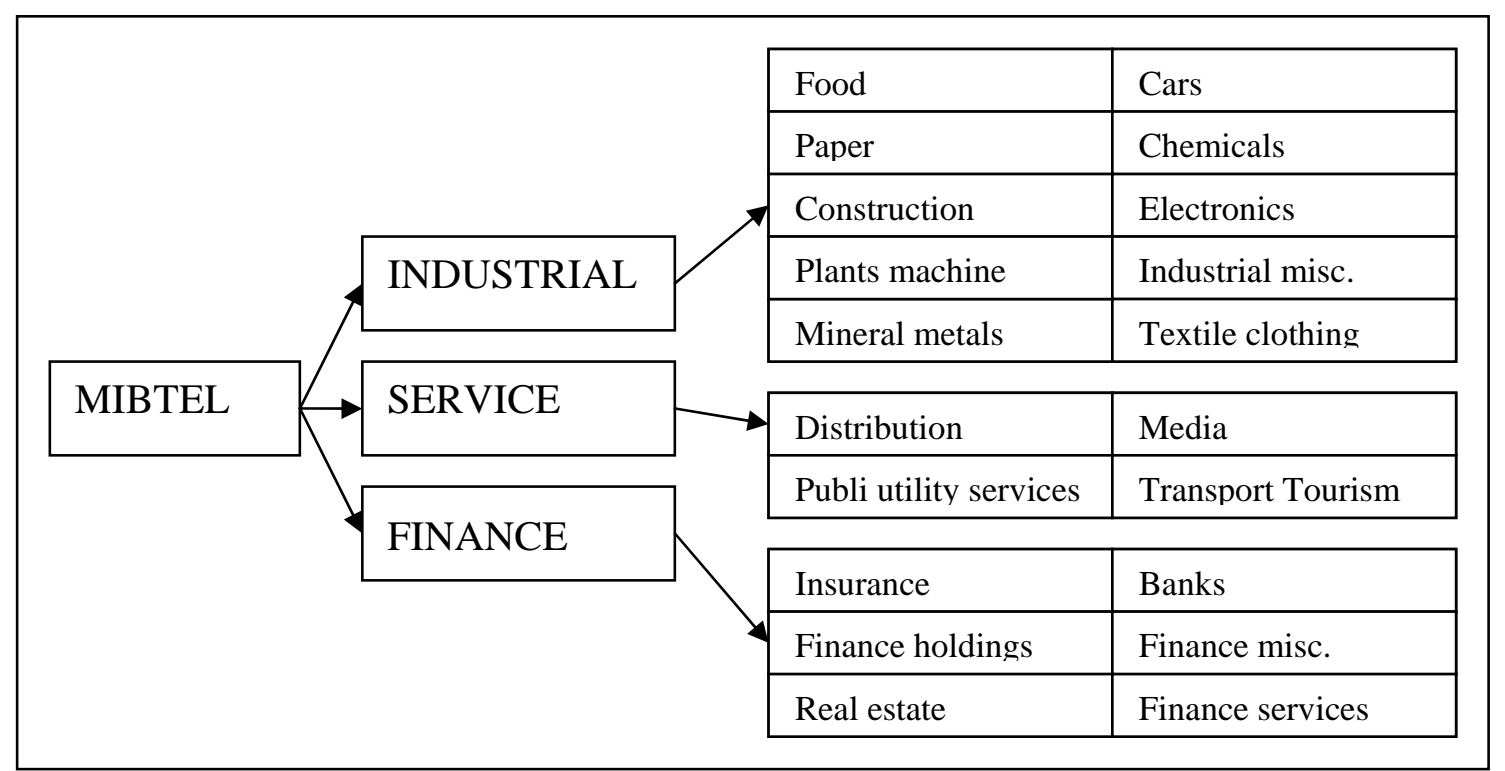

Figure 1: MIBTEL index decomposition by sectors and sub-sectors

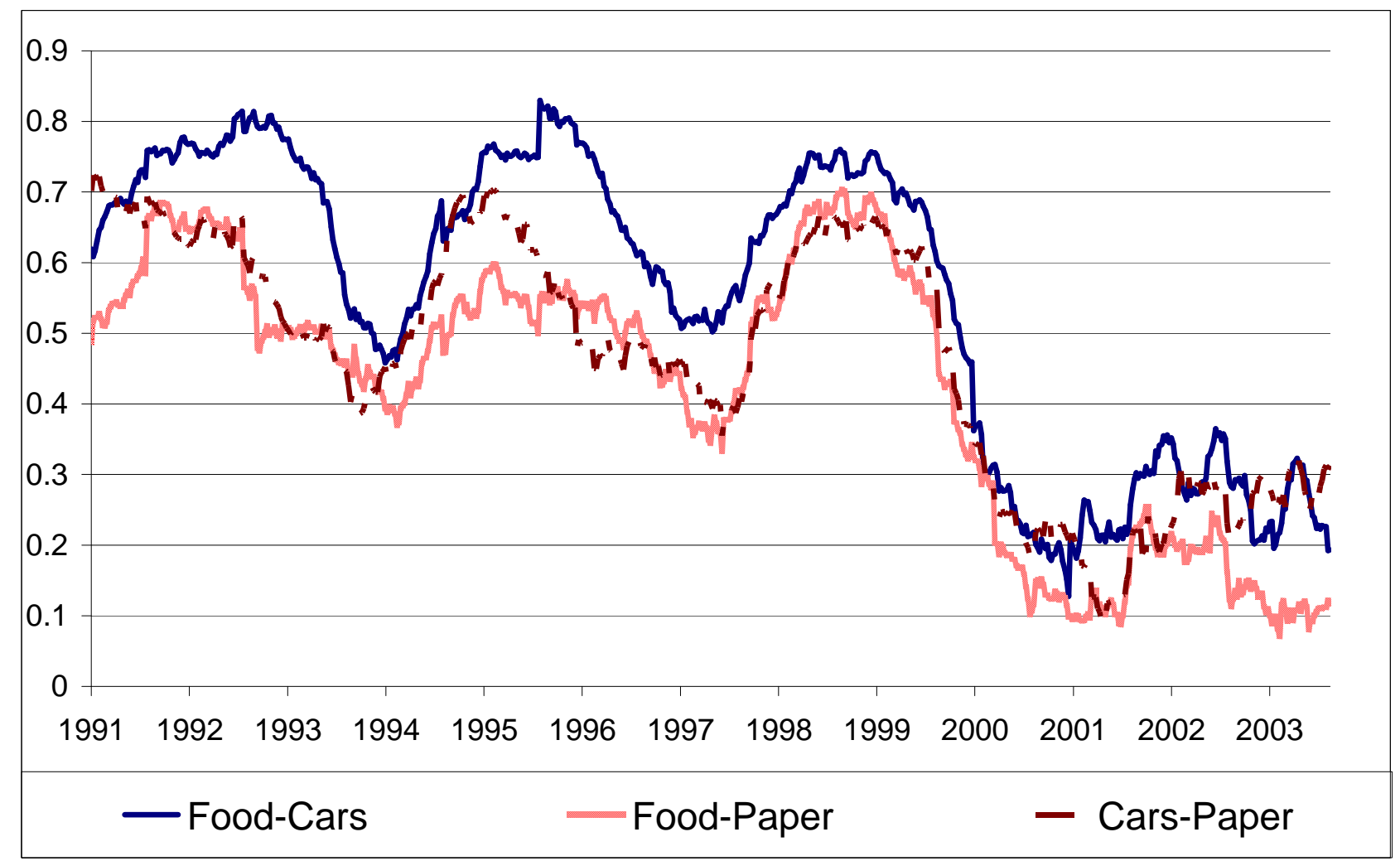

Figure 2: rolling correlations over a 250 observation window for a selected Industrial sub-sectors 


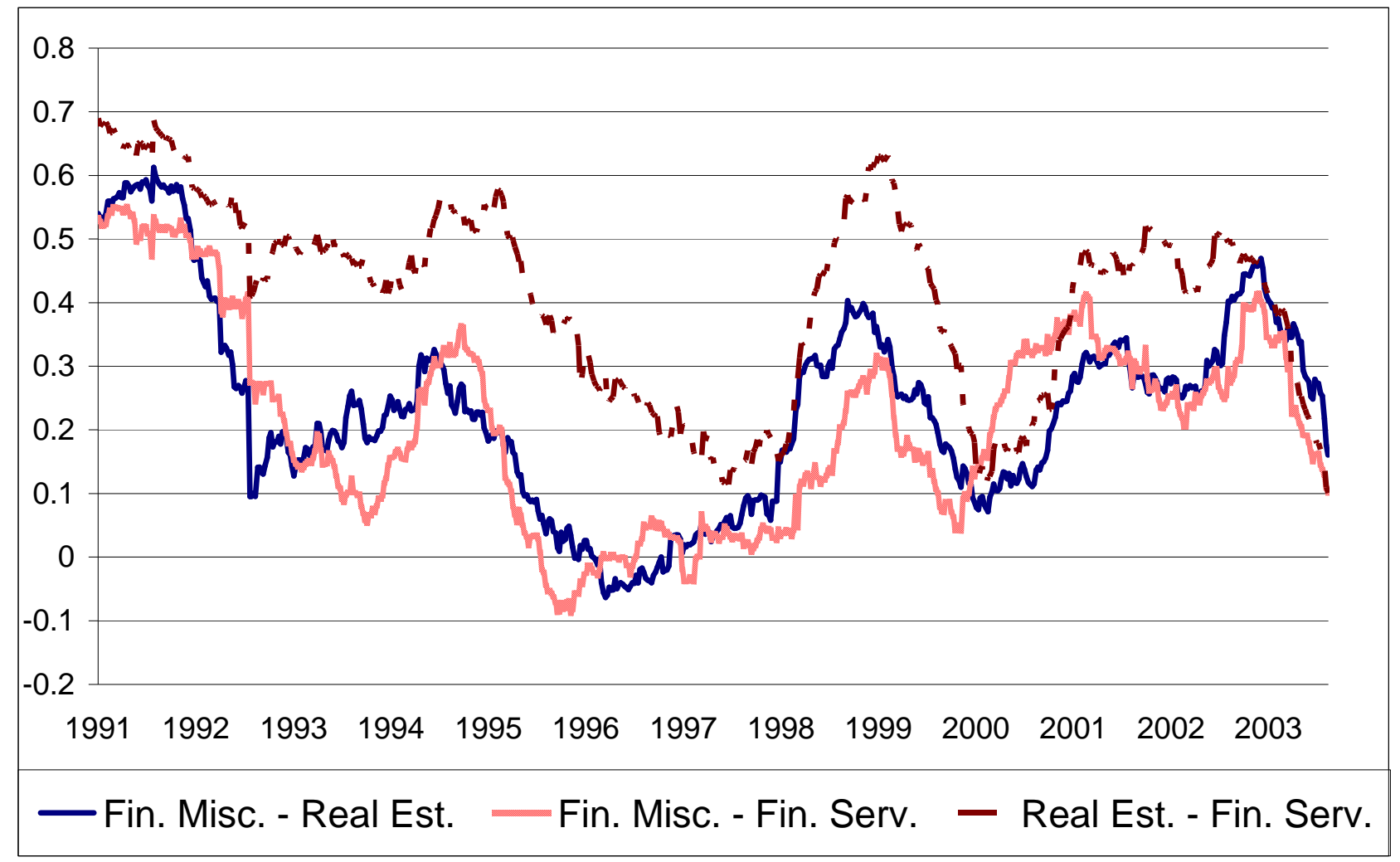

Figure 3: rolling correlations over a 250 observation window for a selected Financial sub-sectors

\begin{tabular}{|c|c|c|c|c|c|c|c|c|c|}
\hline & $\omega$ & $\alpha$ & $\gamma$ & $\beta$ & & $\omega$ & $\alpha$ & $\gamma$ & $\beta$ \\
\hline \multirow{2}{*}{ FOOD } & 0.239 & 0.031 & 0.225 & 0.703 & \multirow{2}{*}{ DISTRIBUTION } & 0.065 & 0.000 & 0.108 & 0.887 \\
\hline & 0.007 & 0.003 & 0.005 & 0.007 & & 0.006 & 0.029 & 0.014 & 0.027 \\
\hline \multirow{2}{*}{ CARS } & 0.068 & 0.031 & 0.127 & 0.883 & \multirow{2}{*}{ MEDIA } & 0.578 & 0.374 & 0.132 & 0.260 \\
\hline & 0.002 & 0.004 & 0.004 & 0.004 & & 0.015 & 0.016 & 0.015 & 0.015 \\
\hline \multirow{2}{*}{ PAPER } & 0.103 & 0.035 & 0.128 & 0.823 & \multirow{2}{*}{ PUB. UTIL. SERV. } & 0.239 & 0.000 & 0.188 & 0.804 \\
\hline & 0.006 & 0.005 & 0.005 & 0.010 & & 0.010 & 0.006 & 0.007 & 0.006 \\
\hline \multirow{2}{*}{ CHEMICALS } & 0.077 & 0.025 & 0.224 & 0.835 & \multirow{2}{*}{$\begin{array}{l}\text { TRANS \& } \\
\text { TOURISM }\end{array}$} & 0.159 & 0.060 & 0.142 & 0.720 \\
\hline & 0.002 & 0.002 & 0.006 & 0.003 & & 0.005 & 0.004 & 0.007 & 0.007 \\
\hline \multirow{2}{*}{ CONSTRUCTION } & 0.099 & 0.011 & 0.251 & 0.783 & \multirow{2}{*}{ INSURANCE } & 0.228 & 0.041 & 0.189 & 0.733 \\
\hline & 0.002 & 0.003 & 0.007 & 0.005 & & 0.135 & 0.034 & 0.044 & 0.129 \\
\hline \multirow[b]{2}{*}{ ELECRONICS } & 0.100 & 0.000 & 0.185 & 0.839 & \multirow{2}{*}{ BANKS } & 0.145 & 0.043 & 0.124 & 0.815 \\
\hline & 0.004 & 0.006 & 0.005 & 0.007 & & 0.007 & 0.005 & 0.005 & 0.007 \\
\hline \multirow{2}{*}{$\begin{array}{l}\text { PLANTS \& } \\
\text { MACHINE }\end{array}$} & 0.154 & 0.067 & 0.350 & 0.652 & \multirow{2}{*}{$\begin{array}{l}\text { FINANCE } \\
\text { HOLDINGS } \\
\end{array}$} & 0.052 & 0.000 & 0.140 & 0.893 \\
\hline & 0.005 & 0.004 & 0.013 & 0.009 & & 0.002 & 0.007 & 0.004 & 0.008 \\
\hline \multirow{2}{*}{$\begin{array}{l}\text { INDUSTRIALS } \\
\text { MISC }\end{array}$} & 0.056 & 0.000 & 0.046 & 0.948 & \multirow{2}{*}{ FINANCE MISC. } & 0.217 & 0.000 & 0.148 & 0.880 \\
\hline & 0.004 & 0.005 & 0.005 & 0.008 & & 0.005 & 0.003 & 0.005 & 0.004 \\
\hline \multirow{2}{*}{$\begin{array}{l}\text { MINERALS } \\
\text { METALS }\end{array}$} & 0.301 & 0.034 & 0.208 & 0.724 & \multirow{2}{*}{ REAL ESTATE } & 0.168 & 0.032 & 0.256 & 0.667 \\
\hline & 0.009 & 0.003 & 0.006 & 0.006 & & 0.006 & 0.005 & 0.008 & 0.010 \\
\hline \multirow{2}{*}{$\begin{array}{l}\text { TEXILE } \\
\text { CLOTHING }\end{array}$} & 0.182 & 0.004 & 0.184 & 0.731 & \multirow{2}{*}{$\begin{array}{l}\text { FINANCE } \\
\text { SERVICES }\end{array}$} & 0.053 & 0.006 & 0.112 & 0.909 \\
\hline & 0.006 & 0.006 & 0.008 & 0.011 & & 0.002 & 0.003 & 0.003 & 0.003 \\
\hline
\end{tabular}

Table 1: GARCH parameter estimates and quasi maximum likelihood standard errors (in italics) for each MIBTEL sub-sector $-\omega$ is the constant of the variance equation, $\alpha$ represents the ARCH term, $\beta$ is the GARCH coefficient and $\gamma$ is the asymmetric effect (the ARCH component is $\alpha+\gamma$ for negative returns and $\alpha$ for positive returns) - we imposed the restrictions $0<\alpha+1 / 2 \gamma+\beta<1$. 


\begin{tabular}{|l|c|c|c|}
\hline Parameters & Estimates & St. Dev. & z-statistics \\
\hline$\alpha$ & 0.021 & 0.005 & 4.196 \\
\hline$\beta$ & 0.489 & 0.199 & 2.455 \\
\hline & \multicolumn{2}{|c|}{ Log Likelihood: -9810.0459 } \\
\hline
\end{tabular}

Table 2: DCC estimates over the full sample with Quasi Maximum Likelihood standard errors

\begin{tabular}{|c|c|c|c|}
\hline Parameters & Estimates & St. Dev. & z-statistics \\
\hline$c_{1}$ & 0.706 & 0.062 & 11.395 \\
\hline$c_{2}$ & 0.991 & 0.193 & 5.134 \\
\hline$c_{3}$ & 0.039 & 0.003 & 12.984 \\
\hline$a_{1}$ & 0.094 & 0.023 & 4.098 \\
\hline$a_{2}$ & 0.065 & 0.034 & 1.904 \\
\hline$a_{3}$ & 0.019 & 0.006 & 3.156 \\
\hline$b_{1}$ & 0.631 & 0.234 & 2.696 \\
\hline$b_{2}$ & 0.977 & 0.009 & 108.595 \\
\hline$b_{3}$ & 0.917 & 0.048 & 19.095 \\
\hline \multicolumn{3}{|c|}{ Log-Likelihood -9320.9534 } \\
\hline
\end{tabular}

Table 3: Diagonal QFDCC estimates over the full sample with Quasi Maximum Likelihood standard errors

\begin{tabular}{|c|c|c|c|}
\hline VaR $\alpha$-level & $1 \%$ & $5 \%$ & $10 \%$ \\
\hline Theoretical & 2.5 & 12.5 & 25 \\
\hline RiskMetrics & 2 & 11 & 18 \\
\hline CCC & 2 & 8 & 13 \\
\hline DCC & 2 & 8 & 13 \\
\hline QFDCC & 3 & 11 & 21 \\
\hline
\end{tabular}

Table 4: Number of exception over last 250 observations for a set of VaR confidence levels

\begin{tabular}{|c|c|c|c|}
\hline VaR Level & $1 \%$ & $5 \%$ & $10 \%$ \\
\hline \multirow{2}{*}{ Model } & \multicolumn{3}{|c|}{ TEST } \\
\cline { 2 - 4 } & \multicolumn{3}{|c|}{ Failure Frequency } \\
\hline RiskMetrics & 0.108 & 0.197 & 2.389 \\
\hline CCC & 0.108 & 1.944 & 7.627 \\
\hline DCC & 0.108 & 1.944 & 7.627 \\
\hline QFDCC & 0.095 & 0.197 & 0.748 \\
\hline & Time Until First Failure \\
\hline RiskMetrics & 0.050 & 1.603 & 0.856 \\
\hline CCC & 0.717 & 2.140 & 1.336 \\
\hline DCC & 0.717 & 2.140 & 1.336 \\
\hline QFDCC & 0.338 & 1.603 & 0.652 \\
\hline
\end{tabular}

Table 5: Tests over VaR exceptions over last 250 observations - the table reports the test statistics which are both asymptotically distributes ad a $\chi^{2}(1)$ - critical levels are 3.84 at the $1 \%$ and 6.63 at the $5 \%$ confidence level. 


\begin{tabular}{|c|c|c|c|}
\hline VaR Level & $1 \%$ & $5 \%$ & $10 \%$ \\
\hline \multirow{2}{*}{ Model } & \multicolumn{3}{|c|}{ LOSS FUNCTIONS } \\
\cline { 2 - 4 } & \multicolumn{3}{|c|}{ Lopez loss function } \\
\hline RiskMetrics & 29.883 & 147.866 & 168.856 \\
\hline CCC & 41.256 & 128.127 & 154.265 \\
\hline DCC & 41.187 & 127.340 & 153.604 \\
\hline QFDCC & 52.075 & 146.091 & 190.107 \\
\hline & \multicolumn{4}{|c|}{ Absolute loss (first order) } \\
\hline RiskMetrics & 172.564 & 140.173 & 110.050 \\
\hline CCC & 182.351 & 154.054 & 127.737 \\
\hline DCC & 182.200 & 153.839 & 127.464 \\
\hline QFDCC & 171.749 & 139.017 & 108.577 \\
\hline & Quadratic loss (second order) \\
\hline RiskMetrics & 595.730 & 474.185 & 426.458 \\
\hline CCC & 661.147 & 514.186 & 451.890 \\
\hline DCC & 660.250 & 513.705 & 451.659 \\
\hline QFDCC & 591.363 & 473.593 & 428.416 \\
\hline & \multicolumn{4}{|c}{ First + Second order loss } \\
\hline RiskMetrics & 768.294 & 614.358 & 536.508 \\
\hline CCC & 843.497 & 668.240 & 579.627 \\
\hline DCC & 842.449 & 667.544 & 579.123 \\
\hline QFDCC & 763.112 & 612.611 & 536.993 \\
\hline
\end{tabular}

Table 6: Loss functions over the 250 observations for the four fitted variance and correlation models

\begin{tabular}{|c|c|c|c|c|}
\hline & RiskMetrics & CCC & DCC & QFDCC \\
\hline \multicolumn{5}{|c|}{ Between VaR Levels } \\
\hline RiskMetrics & 1 & 0.656 & 0.656 & 0.626 \\
\hline CCC & --- & 1 & 0.998 & 0.987 \\
\hline DCC & --- & --- & 1 & 0.987 \\
\hline QFDCC & --- & --- & --- & 1 \\
\hline \multicolumn{5}{|c|}{ Between 1\% VaR exceptions } \\
\hline RiskMetrics & 1 & 0.496 & 0.496 & 0.402 \\
\hline CCC & --- & 1 & 1 & 0.815 \\
\hline DCC & --- & --- & 1 & 0.815 \\
\hline QFDCC & --- & --- & --- & 1 \\
\hline \multicolumn{5}{|c|}{ Between 5\% VaR exceptions } \\
\hline RiskMetrics & 1 & 0.848 & 0.848 & 0.810 \\
\hline CCC & --- & 1 & 1 & 0.848 \\
\hline DCC & --- & --- & 1 & 0.848 \\
\hline QFDCC & --- & --- & --- & 1 \\
\hline \multicolumn{7}{|c|}{ Between 10\% VaR exceptions } \\
\hline RiskMetrics & 1 & 0.701 & 0.701 & 0.697 \\
\hline CCC & --- & 1 & 1 & 0.773 \\
\hline DCC & --- & --- & 1 & 0.773 \\
\hline QFDCC & --- & --- & --- & 1 \\
\hline
\end{tabular}

Table 7: Correlation matrices between VaR levels and exceptions over last 250 observations - CCC and DCC have exactly the same exceptions at all confidence levels 


\begin{tabular}{|c|c|c|c|c|c|}
\hline$\alpha(\%)$ & Theoretical & RiskMetrics & CCC & DCC & QFDCC \\
\hline 1 & 2.5 & 2 & 2 & 2 & 3 \\
\hline 2 & 5 & 7 & 3 & 3 & 6 \\
\hline 3 & 7.5 & 7 & 6 & 6 & 9 \\
\hline 4 & 10 & 8 & 6 & 6 & 9 \\
\hline 5 & 12.5 & 11 & 8 & 8 & 11 \\
\hline 10 & 25 & 18 & 13 & 13 & 21 \\
\hline 15 & 37.5 & 25 & 27 & 26 & 29 \\
\hline 20 & 50 & 37 & 31 & 31 & 37 \\
\hline 25 & 62.5 & 47 & 43 & 43 & 45 \\
\hline 30 & 75 & 57 & 52 & 52 & 58 \\
\hline
\end{tabular}

Table 8: The table reports the theoretical and empirical number of exceptions for several confidence levels for the fitted models 